\title{
ARTICLE OPEN The role of quantum coherence in non-equilibrium entropy production
}

\author{
Jader P. Santos $\mathbb{D}^{1}$, Lucas C. Céleri ${ }^{2}$, Gabriel T. Landi $\mathbb{D}^{1}$ and Mauro Paternostro ${ }^{3}$
}

Thermodynamic irreversibility is well characterized by the entropy production arising from non-equilibrium quantum processes. We show that the entropy production of a quantum system undergoing open-system dynamics can be formally split into a term that only depends on population unbalances, and one that is underpinned by quantum coherences. This allows us to identify a genuine quantum contribution to the entropy production in non-equilibrium quantum processes. We discuss how these features emerge both in Lindblad-Davies differential maps and finite maps subject to the constraints of thermal operations. We also show how this separation naturally leads to two independent entropic conservation laws for the global system-environment dynamics, one referring to the redistribution of populations between system and environment and the other describing how the coherence initially present in the system is distributed into local coherences in the environment and non-local coherences in the systemenvironment state. Finally, we discuss how the processing of quantum coherences and the incompatibility of non-commuting measurements leads to fundamental limitations in the description of quantum trajectories and fluctuation theorems.

npj Quantum Information (2019)5:23; https://doi.org/10.1038/s41534-019-0138-y

\section{INTRODUCTION}

Irreversible processes undergone by an open quantum system are associated with a production of entropy that is fundamentally different from the possible flow of entropy resulting from the mutual coupling of the system with its environment. Such inevitable contribution to the entropy change of the state of a system is termed entropy production. If entropy is labelled as $S$, we describe its rate of change as

$\frac{\mathrm{d} S}{\mathrm{~d} t}=\Pi-\Phi$,

where $\Phi$ is the entropy flux rate from the system to the environment and $\Pi$ is the entropy production rate. According to the second law of thermodynamics, we should have $\Pi \geq 0$, with $\Pi$ $=0$ if and only if the system is at equilibrium. The characterization of the degree of irreversibility of a process, and thus the understanding of entropy production is both fundamentally relevant and technologically desirable. On one hand, such understanding would provide the much needed foundations to the emergence of time-symmetry breaking entailed by irreversibility and epitomized, for instance, by seminal results such as Onsager's theory of irreversible currents. ${ }^{1-5}$ On the other hand, a characterization of irreversible entropy could help us designing thermodynamically efficient quantum technologies. ${ }^{6,7}$

In general, the open dynamics of a quantum system gives rise to two processes. The first corresponds to the transitions between energy levels of the system, which will cause the populations to adjust to the values imposed by the bath. Measures of the entropy production for this kind of processes have been known for many years, particularly in the context of Fokker-Planck equations ${ }^{8-10}$ and Pauli master equations. ${ }^{11,12}$
The second process undergone by an open quantum system is the loss of coherence in the energy eigenbasis. Coherence is an essential resource for quantum processes, ${ }^{13}$ likely representing the ultimate feature setting quantum and classical worlds apart. Only very recently have steps towards the formulation of a unified resource theory of coherence been made. ${ }^{14}$ While the role of quantum coherences in thermodynamics is yet to be fully understood (both qualitatively and quantitatively), it is known that coherence plays a role in the allowed transitions generated by thermal operations. ${ }^{15-21}$ Moreover, it also affects the performance of non-adiabatic work protocols. $22-25$

Understanding the interplay between population dynamics and loss of coherence represents a pressing problem in the field of thermodynamics of quantum systems. In particular, one is naturally led to wonder how entropy production is underpinned by the dynamics of quantum coherences in irreversible open dynamics.

Shedding light on such an intimate relation is the main motivation of this paper, where we put forward a formal description of entropy production in terms of two contributions, one clearly related to the dynamics of populations and the other depending explicitly on the coherence within the state of the system undergoing the open process. We first discuss how these features emerge in the case of Davies-type master equations, for which the formulation is transparent. We then address more general dynamical maps satisfying the constraints of thermal operations. $^{26}$ In this case, we show how our result can be connected to recent resource theoretical developments. ${ }^{17,19}$

Afterwards, we address the main physical implications of these two contributions to the entropy production. First, we discuss how it enables one to construct two independent entropic conservation laws for the global system-environment dynamics. The first is

\footnotetext{
${ }^{1}$ Instituto de Física da Universidade de São Paulo, 05314-970 São Paulo, Brazil; ${ }^{2}$ Instituto de Física, Universidade Federal de Goiás, Caixa Postal 131, 74001-970 Goiânia, Brazil and ${ }^{3}$ Centre for Theoretical Atomic, Molecular and Optical Physics, School of Mathematics and Physics, Queen's University Belfast, Belfast BT7 1NN, UK Correspondence: Gabriel T. Landi (gtlandi@if.usp.br)
}

Received: 18 June 2018 Accepted: 9 January 2019

Published online: 01 March 2019 
entirely classical and relates to the redistribution of populations between system and enviroment. The second, on the other hand, dictates how the coherence initially prepared in the system is distributed among local coherences in the environment and nonlocal coherences in the global system-environment state. Lastly, we address the issue of how to access entropy production by means of quantum measurements and quantum trajectories. We show that even in the presence of coherence, it is possible to construct measurement protocols, which satisfy fluctuation theorems. However, unlike in the case of closed systems, ${ }^{25}$ it is not possible to consider a single measurement protocol in which the fluctuation theorems are satisfied for both contributions of the entropy production individually.

Several advances in the past decade have consistently shown that it is possible to engineer systems in which thermodynamics coexists with quantum effects. It is our hope that the framework put forth in this paper may contribute for the development of a unified theory describing both thermal and quantum resources.

\section{RESULTS}

Entropy production due to the processing of coherence

Coherence is a basis-dependent concept and, in principle, no preferred basis exists. Here we adopt the viewpoint according to which a preferred basis only emerges when it is imposed by the environment, ${ }^{27,28}$ a perspective that is typically referred to as einselection. There are several scenarios in which a preferred basis may emerge. We shall focus on two of them. The first are Davies maps, ${ }^{29-31}$ which make use of the weak-coupling approximation, and the second are the so-called thermal operations. ${ }^{17,19,26}$ Both scenarios lead to energy conservation so that the energy eigenbases are naturally selected as preferred bases. However, while in the weak-coupling scenario, energy conservation is taken as an approximation midway through the calculations, in the case of thermal operations this is imposed from the start, as an assumption of the model. Consequently, thermal operations are much more versatile, while simultaneously offering a much clearer physical interpretation. We also assume, for simplicity, that the energy eigenvalues of the system are non-degenerate, since this would cause the preferred basis to depend on additional details of the system-environment interaction, which one seldom has access to.

Lindblad-Davies maps. We begin by analyzing the so-called Lindblad-Davies maps, which generally describe the weak contact of a system with a thermal environment. They have the form ${ }^{29-31}$

$\frac{\mathrm{d} \rho_{S}}{\mathrm{~d} t}=-i\left[H_{S}, \rho_{S}\right]+D\left(\rho_{S}\right)$,

where $\rho_{S}$ is the density matrix of the system, $H_{S}$ is the Hamiltonian and $D\left(\rho_{S}\right)$ is a Lindblad dissipator having the Gibbs state $\rho_{S}^{\text {eq }}=$ $e^{-\beta H_{s}} / Z_{S}$ as a fixed point; i.e. $D\left(\rho_{S}^{\mathrm{eq}}\right)=0$ (here $Z_{S}=t r e^{-\beta H_{s}}$ is the partition function and $\beta$ the inverse temperature of the bath).

Davies maps are known to lead to a separation between the evolution of populations, $p_{n}=\left\langle n\left|\rho_{S}\right| n\right\rangle$, of the density matrix and the off-diagonal coherences $p_{n m}=\left\langle n\left|\rho_{s}\right| m\right\rangle$. Here we have called $\{\mid$ $n\rangle$ \} the elements of the basis imposed by the specific environment being considered. In the specific case of a Davies map, such basis is that of the energy eigenstates, whose set of eigenvalues we call $\left\{E_{n}\right\}$.

Following Eq. (2), the populations evolve following the Pauli master equation

$$
\frac{\mathrm{d} p_{n}}{\mathrm{~d} t}=\sum_{m}\left[W(n \mid m) p_{m}-W(m \mid n) p_{n}\right]
$$

where $W(n \mid m)$ are the transition rates from the energy level $E_{n}$ to level $E_{m}$. They satisfy the detailed-balance condition

$\frac{W(n \mid m)}{W(m \mid n)}=\frac{p_{n}^{\mathrm{eq}}}{p_{m}^{\mathrm{eq}}}=e^{-\beta\left(E_{n}-E_{m}\right)}$,

where $p_{n}^{\mathrm{eq}}=\left\langle n\left|\rho_{S}^{\mathrm{eq}}\right| n\right\rangle$. As for the coherences, provided that the Bohr frequencies $\omega_{n m}=E_{n}-E_{m}$ are non-degenerate, they evolve independently of each other according to the equation

$$
\frac{\mathrm{d} p_{n m}}{\mathrm{~d} t}=-\left\{i \omega_{n m}-\frac{1}{2} \sum_{k}[W(k \mid n)+W(k \mid m)]\right\} p_{n m} \text {. }
$$

As the second term in the right-hand side can be seen as an effective (generally temperature-dependent) damping term, the evolution of the coherences can be interpreted as that resulting from damped oscillations.

We now define the non-equilibrium free energy as

$F\left(\rho_{S}\right)=\operatorname{tr}\left(H_{S} \rho_{S}\right)+T \operatorname{tr}\left(\rho_{S} \ln \rho_{S}\right)$,

where $T$ is the temperature of the bath. While Eq. (6) reduces to the usual expression $F_{\text {eq }}=-T$ In $Z_{S}$ at equilibrium, for general non-equilibrium states, we can write

$$
F\left(\rho_{S}\right)=F_{\text {eq }}+T S\left(\rho_{S} \| \rho_{S}^{\mathrm{eq}}\right) \text {, }
$$

where $S(\rho \| \sigma)=\operatorname{tr}(\rho \ln \rho-\rho \ln \sigma)$ is the quantum relative entropy. As $S\left(\rho_{S}|| \rho_{S}^{\text {eq }}\right) \geq 0$, we have that $F\left(\rho_{S}\right) \geq F_{\text {eq. }}$. This condition thus defines the equilibrium state of a system as the state that minimizes the free energy. ${ }^{32}$ Moreover, it establishes that during relaxation, the free energy is a monotonically decreasing function of time whose value is determined by the distance, in state space, between the instantaneous state of the system $\rho_{S}$ and its equilibrium version $\rho_{s}^{\text {eq }}$. Hence, one is naturally led to define the entropy production rate as ${ }^{30,33-36}$

$\Pi=-\frac{1}{T} \frac{\mathrm{d} F\left(\rho_{S}\right)}{\mathrm{d} t}$

which ensures that $\Pi \geq 0$ and $\Pi=0$ iff $\rho_{S}=\rho_{S}^{\text {eq }}$.

Next we notice that in terms of the eigenbasis of $H_{S}$, we can always split $S\left(\rho \| \rho_{\text {eq }}\right)$ as

$S\left(\rho_{S} \| \rho_{S}^{\mathrm{eq}}\right)=S\left(p_{S} \| p_{S}^{\mathrm{eq}}\right)+C\left(\rho_{S}\right)$.

Here $\mathcal{S}\left(p_{S} \| p_{S}^{\mathrm{eq}}\right)=\sum_{n} p_{n} \ln p_{n} / p_{n}^{\mathrm{eq}}$ is the Kullback-Leibler divergence of the classical probability distribution entailed by the populations $p_{S}=\left\{p_{n}\right\}$ from that of the equilibrium state $p_{S}^{\text {eq }}=\left\{p_{n}^{\text {eq }}\right\}$. Moreover, we have introduced the relative entropy of coherence ${ }^{14}$

$C\left(\rho_{S}\right)=S\left(\Delta_{H_{S}}\left(\rho_{S}\right)\right)-S\left(\rho_{S}\right)$,

where $\Delta_{H_{S}}(\rho)$ is the dephasing map, acting on the density matrix $\rho_{S}$, which removes all coherences from the various energy eigenspaces of $H_{S}$. With this at hand, Eq. (7) becomes

$F\left(\rho_{S}\right)=F_{\text {eq }}+T S\left(p_{S} \| p_{S}^{\text {eq }}\right)+T C\left(\rho_{S}\right)$.

This is one of the central results of this work: it shows that quantum coherence is actually a part of the non-equilibrium free energy, and thus contributes significantly to the determination of the non-equilibirum thermodynamics stemming from Eq. (2). The second term in $F\left(\rho_{s}\right)$ quantifies the increase in free energy due to population imbalance with respect to the equilibrium configuration and, as such, is a purely classical term. The last term, which is of a genuine quantum nature, determines the surplus in free energy that a non-equilibrium state with quantum coherences offers with respect to its diagonal (and thus classical) counterpart.

Let us now use the formal splitting in Eq. (11) to recast the 
entropy production rate in Eq. (8) as

$\Pi=\Pi_{d}+\Upsilon$.

The first term is written as

$$
\begin{aligned}
\Pi_{d} & =-\frac{\mathrm{d}}{\mathrm{d} t} S\left(p_{S} \| p_{S}^{\mathrm{eq}}\right) \\
& =\frac{1}{2} \sum_{n, m}\left[W(n \mid m) p_{m}-W(m \mid n) p_{n}\right] \ln \frac{W(n \mid m) p_{m}}{W(m \mid n) p_{n}} .
\end{aligned}
$$

and is then exactly the classical result derived in refs. ${ }^{11,12}$ The second contribution reads

$$
\Upsilon=-\frac{\mathrm{d} C\left(\rho_{S}\right)}{\mathrm{d} t}
$$

which thus shows that the rate of loss of coherence that might ensue from the dynamics of the system enters quantitatively into the entropy production: the rate at which entropy is produced in a process where quantum coherences are destroyed as a result of the coupling with an environment surpasses the corresponding classical version. Clearly both $\Pi_{d}$ and $\gamma$ are non-negative and null only for $\rho_{S}=\rho_{S}^{\text {eq }}$.

Finally, let us address the entropy flux defined in Eq. (1). Using Eq. (8) we find

$\Phi=\frac{\Phi_{E}}{T}=-\frac{1}{T} \sum_{n} E_{n}^{S} \frac{\mathrm{d} p_{n}}{\mathrm{~d} t}$,

where $\Phi_{E}$ is the energy flux from the system to the environment. In deriving this equation we have used $p_{n}^{\mathrm{eq}}=\exp \left[-\beta E_{n}^{S}\right] / Z_{s}$. The entropy flux has thus no contribution arising from quantum coherences: entropy (and energy) will only flow due to imbalances in the populations. Any loss of coherence contributes only to the entropy production rate and has no associated flux. It is important to emphasize that this is a feature of the present type of master equation. In other situations, such as strong coupling dynamics, the coherences in the system may play a relevant role in the entropy and heat fluxes.

Thermal operations. We now address the case of more general maps with the aim of gaining access to the environmental degrees of freedom, hence enhancing our understanding of the two contributions to the entropy production from the perspective of the joint system-environment properties.

We consider explicitly thermal operations, which have been the subject of numerous recent investigations in the context of resource theories. ${ }^{17,19,26} \mathrm{~A}$ thermal operation is physically described as the interaction of the system with an arbitrary environment, initially prepared in equilibrium $\rho_{E}^{\text {eq }}=e^{-\beta H_{E}} / Z_{E}$, through a unitary $U$, which conserves the total energy, that is an operation such that $\left[U, H_{S}+H_{E}\right]=0$. In this sense, the thermal operation hypothesis reminds us of the framework set by the weak-coupling approximation. However, it allows us to go significantly beyond the limitations of weak-coupling, and thus extend our analysis to a larger set of physically meaningful cases. $^{37}$ We also call attention to the fact that in thermal operations the energy conservation condition is only imposed on the global unitary $U$, irrespective of how this is generated. One way to do so is by means of a time-dependent interaction that is turned on and off. Another, much simpler approach is to simply have any potential $V$, which satisfies $\left[V, H_{S}+H_{E}\right]=0$. This will then generate a time-independent unitary $U=e^{-\left(H_{S}+H_{E}+V\right) t}$, which will be energy conserving. This type of thermal operation was recently used in ref. ${ }^{38}$ to study the heat exchange between two qubits in a magnetic resonance setup.

The state of the composite system after the evolution in a thermal operation will be

$\rho_{S E}^{\prime}=U\left(\rho_{S} \otimes \rho_{E}^{\mathrm{eq}}\right) U^{\dagger}$.
We label the environmental energies and eigenstates as $\left\{E_{\mu}^{E}\right\}$ and $\{|\mu\rangle\}$, respectively. We also call $q_{\mu}=e^{-\beta E_{\mu}^{E}} / Z_{E}$ its initial thermal populations. Energy conservation then implies that

$$
\langle m, \nu|U| n, \mu\rangle \propto \delta\left(E_{n}^{S}+E_{\mu}^{E}-E_{m}^{S}-E_{\nu}^{E}\right) .
$$

Tracing out the environment one obtains the Kraus map for the system

$\rho_{S}^{\prime}=\operatorname{tr}_{E}\left[U\left(\rho_{S} \otimes \rho_{E}^{\mathrm{eq}}\right) U^{\dagger}\right]=\sum_{\mu, \nu} M_{\mu, \nu} \rho_{S} M_{\mu, \nu}^{\dagger}$

where $M_{\mu, \nu}=\sqrt{q_{\mu}}\langle\nu|U| \mu\rangle$. Clearly, the Gibbs state $\rho_{S}^{\text {eq }}$ is a fixed point of this equation. Moreover, the Davies maps studied in the previous section correspond to particular Markovian limits of Eq. (18).

The energy conservation condition implies that when the eigenvalues of $H_{S}$ are non-degenerate, Eq. (18) is an incoherent operation in the sense of ref. ${ }^{14}$ That is, defining the energy eigenstates as the set of incoherent states, this process always maps incoherent states into incoherent states. This in turn allows for an independent processing of both populations and coherences. The diagonal entries will, in particular, evolve according to the classical Markov chain

$p_{m}^{\prime}=\sum_{n} Q(m \mid n) p_{n}$,

where $Q(m \mid n)=\sum_{\mu, \nu}\left|\left\langle m\left|M_{\mu, \nu}\right| n\right\rangle\right|^{2}$ is the transition probability from state $n$ to state $m$, a quantity playing the role of the transition rate $W(m \mid n)$ in Eq. (3). The processing of the coherences, on the other hand, takes place independently of the changes in populations. In particular, if the Bohr frequencies $\omega_{m n}$ are nondegenerate, this processing takes the simple form

$p_{n, m}^{\prime}=a_{n, m} p_{n, m}, \quad a_{n, m}=\sum_{\mu, \nu}\left\langle n\left|M_{\mu, \nu}\right| n\right\rangle\left\langle m\left|M_{\mu, \nu}^{\dagger}\right| m\right\rangle$.

As shown in ref. ${ }^{19}$ the processing of coherence is not independent of the population changes, but must satisfy the inequality

$\left|a_{n, m}\right|^{2} \leq Q(n \mid n) Q(m \mid m)$,

which provides a bound to the maximum amount of coherence that may be lost in a thermal operation.

We now turn to the analysis of the entropy production in this scenario. Unlike the previous section, as the dynamics in Eq. (18) is in general non-Markovian and we only have access to the global map, it is not possible to address the rate of entropy production $\Pi$, but only the total entropy $\Sigma$ produced in the process. In this case, using the contractive property of the relative entropy, ${ }^{39}$ we have $S\left(\rho_{S}^{\prime} \| \rho_{S}^{\text {eq }}\right) \leq S\left(\rho_{S} \| \rho_{S}^{\text {eq }}\right)$. Consequently, the free energy Eq. (7) remains a non-increasing function, thus justifying the following definition of total entropy production

$\Sigma=-\frac{\Delta F}{T}=S\left(\rho_{S} \| \rho_{S}^{\mathrm{eq}}\right)-S\left(\rho_{S}^{\prime} \| \rho_{S}^{\mathrm{eq}}\right) \geq 0$

This expression may be taken as a basic postulate in our framework, motivated by physical consistency arguments that can be even reinforced by a quantum trajectory point of view, as discussed e.g. in refs. ${ }^{40,41}$ Other approaches have also been used elsewhere. ${ }^{42,43}$

As $\Sigma$ is also based on the quantum relative entropy, a splitting akin to Eq. (12) into non-negative population-related and a 
coherence-related terms is in order, and we have

$\Sigma=\Sigma_{d}+\equiv$,

where

$\Sigma_{d}=S\left(p_{S} \| p_{S}^{\mathrm{eq}}\right)-S\left(p_{S}^{\prime} \| p_{S}^{\mathrm{eq}}\right)$

$\Xi=\mathcal{C}\left(p_{S}\right)-\mathcal{C}\left(p_{S}^{\prime}\right)$

The non-negativity of $\Sigma_{d}$ follows immediately from the fact that in thermal operations diagonal elements evolve independently of coherences. The positivity of $\equiv$, on the other hand, follows from the fact that a thermal operation is incoherent. ${ }^{14}$ In the limit where the Davies maps are recovered, $\Sigma_{d}$ and $\equiv$ become respectively the integrated versions of $\Pi_{d}$ and $\gamma$ in Eq. (12). This demonstrates the generality, under suitable and reasonable assumptions on the form of the system-environment coupling, of the central result of our investigation.

Implications of the central results

We now explore which considerations can be drawn in light of the formal splitting of the entropy production demonstrated above.

Entropic conservation laws. The structure of thermal operations and Eq. (16) imply a series of conservation rules for the processing of populations and coherences. First, energy conservation implies that the total entropy production in Eq. (22) can be written as $\mathrm{s}^{42,44}$

$\Sigma=S\left(\rho_{E}^{\prime} \| \rho_{E}^{\mathrm{eq}}\right)+\mathcal{I}\left(\rho_{S E}^{\prime}\right)$,

where $\rho_{E}^{\prime}=\operatorname{tr}_{S}\left(\rho_{S E}^{\prime}\right)$, and $\mathcal{I}\left(\rho_{A B}\right)=S\left(\rho_{A}\right)+S\left(\rho_{B}\right)-S\left(\rho_{A B}\right)$ is the mutual information of a bipartite system. This gives an interesting interpretation of $\Sigma$ as being related to the change in the environmental state, measured by the first term, and the total degree of correlations created by the thermal operation, measured by the mutual information. As discussed in ref. ${ }^{44}$ Eq. (26) also provides a clear interpretation of how irreversibility emerges from a global unitary dynamics, ascribing it to two reasons. One is the creation of correlations between system and environment, which are never recovered once one traces out the environment (hence giving rise to an irretrievable loss of information). The second is related to the fact that the system pushes the environment away from equilibrium. As shown in ref. ${ }^{40}$ if $\rho_{E}^{\prime}-\rho_{E}^{\mathrm{eq}} \sim \varepsilon$ then $S\left(\rho_{E}^{\prime} \| \rho_{E}^{\mathrm{eq}}\right) \sim \varepsilon^{2}$, whereas $S\left(\rho_{E}^{\prime}\right)-S\left(\rho_{E}^{\mathrm{eq}}\right) \sim \varepsilon$. Thus, when the reservoir is large, the first term becomes negligible and the main contribution to the entropy production will come from the total correlations created between system and environment.

Next we note that as the map (16) is unitary, it follows that $S\left(\rho_{S E}^{\prime}\right)=S\left(\rho_{S E}\right)$. However, as $\left[U, H_{S}+H_{E}\right]=0$, the dephasing operation $\Delta_{H_{S}+H_{E}}$ commutes with the unitary evolution so that in addition to the total entropy being conserved, the same is also true for the dephased entropies

$S\left(\Delta_{H_{S}+H_{E}}\left(\rho_{S E}^{\prime}\right)\right)-S\left(\Delta_{H_{S}}\left(\rho_{S}\right)\right)+S\left(\rho_{E}^{\mathrm{eq}}\right)$

This result reflects how the changes in population in the system and environment affect the information content of the diagonal elements of $\rho_{S E}^{\prime}$. Note that the left-hand side contemplates, at most, coherences in the degenerate subspaces of $H_{S}+H_{E}$, which are not resources from the perspective of this operation.

From Eqs. (26) and (27), it follows that a similar law must also hold for the relative entropy of coherence

$C\left(\rho_{S E}^{\prime}\right)=C\left(\rho_{S}\right)$.

where $C\left(\rho_{S E}^{\prime}\right)=S\left(\Delta_{H_{S}+H_{E}}\left(\rho_{S E}^{\prime}\right)\right)-S\left(\rho_{S E}^{\prime}\right)$. Thus, we see that the reduction in the coherence of the system after the map is due to a redistribution of this coherence over the global system- environment state.

Substituting Eq. (28) into Eq. (25) shows that the contribution of the entropy production due to quantum coherences may be written as

$\Xi=C\left(\rho_{S E}^{\prime}\right)-C\left(\rho_{S}^{\prime}\right)$.

Thus, the entropy production due to quantum coherences can be seen as the mismatch between the global coherences in the correlated system-environment state and the local coherences in the final state. We can also relate $\equiv$ to the notion of correlated coherence, introduced recently in ref. ${ }^{45}$ and defined as

$C_{\mathrm{cc}}\left(\rho_{S E}^{\prime}\right)=C\left(\rho_{S E}^{\prime}\right)-C\left(\rho_{S}^{\prime}\right)-C\left(\rho_{E}^{\prime}\right) \geq 0$,

where $C\left(\rho_{E}^{\prime}\right)=S\left(\Delta_{H_{E}}\left(\rho_{E}^{\prime}\right)\right)-S\left(\rho_{E}^{\prime}\right)$. This quantity therefore represents the portion of coherence that is contained within the correlations between system and environment. Combining the Eqs. (29) and (30), it is then possible to write

$\Xi=C\left(\rho_{E}^{\prime}\right)+C_{\mathrm{cc}}\left(\rho_{S E}^{\prime}\right)$.

This has the same form as Eq. (26), with the first term representing the local coherences developed in the environment and the second term representing the non-local contribution. Thus, we may conclude from this result that entropy production has a clearly local contribution, related to the creation of coherences in the environment, and a non-local contribution related to the creation of shared coherences in the systemenvironment state.

Stochastic trajectories and fluctuation theorems. Lastly, let us consider the stochastic version of the entropy production arising from quantum trajectories. In order to correctly treat the coherences present in the system, we adopt the following procedure. In the forward protocol, the environment is prepared in the thermal state $\rho_{E}=\sum_{\mu} q_{\mu}|\mu\rangle\langle\mu|$, where $q_{\mu}=e^{-\beta E_{\mu}^{E}} / Z_{E}$. The system, on the other hand, is taken to be in an arbitrary state $\rho_{S}=\sum_{a} p_{a}\left|\psi_{a}\right\rangle\left\langle\psi_{a}\right|$, which in general contain coherences, so that the basis $\left|\psi_{a}\right\rangle$ is incompatible with the energy basis $|n\rangle$. As the first step in the protocol, we then perform local measurements in the bases $\left|\psi_{a}\right\rangle$ and $|\mu\rangle$ of $S$ and $E$, obtaining the state $\left|\psi_{a}, \mu\right\rangle$ with probability $p_{\alpha} q_{\mu}$. Next, we evolve both with a joint unitary $U$. Finally, in the third step we measure only the environment, again in the energy basis $|v\rangle$. Due to the measurement backaction, the state of the system then collapses to the pure state

$$
\left|\Phi_{F \mid a \mu \nu}\right\rangle=\frac{\left\langle\nu|U| \psi_{a}, \mu\right\rangle}{\sqrt{P_{F}(\nu \mid a \mu)}}
$$

where $P_{F}(v \mid a \mu)=\left\|\left\langle v|U| \psi_{a}, \mu\right\rangle\right\|^{2}$. For a discussion on the effects of choosing different bases for the second measurement in the environment, see ref. ${ }^{40}$

The final state $\rho_{s}^{\prime}$ of the system will then be given by an ensemble average over all possible final states (32), weighted by the probability of the stochastic trajectory $(a, \mu, v)$; viz.,

$\rho_{S}^{\prime}=\sum_{a, \mu, \nu} P_{F}(\nu \mid a, \mu) p_{a} q_{\mu}\left|\Phi_{F \mid a \mu \nu}\right\rangle\left\langle\Phi_{F \mid a \mu \nu}\right|$

One may directly verify that this state is indeed equal to the unmeasured final state $\rho_{S}^{\prime}$ in Eq. (18). The states (32), however, are not the eigenstates of $\rho_{S}^{\prime}$ and in fact don't even form a basis. The diagonal structure of $\rho_{S}^{\prime}$ will thus be of the form $\rho_{S}^{\prime}=\sum_{\beta} p_{\beta}^{\prime}\left|\psi_{\beta}^{\prime}\right\rangle\left\langle\psi_{\beta}^{\prime}\right|$, where $\left|\psi_{\beta}^{\prime}\right\rangle$ is a new basis set that is not trivially related to neither $\left|\psi_{a}\right\rangle$ nor $\left|\Phi_{F \mid a \mu \nu}\right\rangle$. This is the key difference that appears due to the presence of coherences (if the initial state of the system were diagonal, the same would be true 
for the final state, since this is a thermal operation). The probabilities $p_{\beta}^{\prime}$ will be given by

$p_{\beta}^{\prime}=\sum_{a, \mu, \nu} p_{\beta \mid \alpha, \mu, \nu} P_{F}(\nu \mid a, \mu) p_{\alpha} q_{\mu}$,

where $p_{\beta \mid a, \mu, \nu}=\left|\left\langle\psi_{\beta}^{\prime} \mid \Phi_{F \mid a \mu \nu}\right\rangle\right|^{2}$ is the conditional probability of finding the system in $\left|\psi_{\beta}^{\prime}\right\rangle$ given that it ended the forward protocol in $\left|\Phi_{F \mid a \mu v}\right\rangle$.

The stochastic trajectory generated by the measurement outcomes is specified by the three quantum numbers $(a, \mu, v)$. However, following ref. ${ }^{46}$ we may augment the trajectory (an idea first introduced by Dirac ${ }^{47}$ ) by introducing $\beta$ as an additional quantum number, so that the trajectory $\mathcal{X}=(a, \mu, \beta, \nu)$ is defined by the probability

$\mathcal{P}_{F}[\mathcal{X}]=p_{\beta \mid a, \mu, \nu} P_{F}(\nu \mid a, \mu) p_{a} q_{\mu}$.

Indeed, using the definition (32), one readily finds that

$p_{\beta \mid a, \mu, \nu} P_{F}(\nu \mid a, \mu)=\left|\left\langle\psi_{\beta}^{\prime}, \nu|U| \psi_{\alpha}, \mu\right\rangle\right|^{2}=P(\beta, \nu \mid a, \mu)$,

which is nothing but the transition probability of observing a transition from $\left|\psi_{a}, \mu\right\rangle$ to $\left|\psi_{\beta}^{\prime}, \nu\right\rangle$.

Next, we define the backward protocol. The initial state of the system is drawn from one of the possible eigenstates $\left|\psi_{\beta}^{\prime}\right\rangle$ of $\rho_{S}^{\prime 48}$ whereas the environment is taken to be in equilibrium and is again measured in the energy basis $|v\rangle$. This yields the state $\left|\psi_{\beta}^{\prime}, \nu\right\rangle$ with probability $p_{\beta}^{\prime} q_{\nu}$. We then apply the time-reversed unitary $U^{\dagger}$ and, in the end, measure $E$ in the basis $|\mu\rangle$. As a consequence the system collapses to

$\left|\Phi_{B \mid \beta \nu \mu}\right\rangle=\frac{\left\langle\mu\left|U^{\dagger}\right| \psi_{\beta}^{\prime}, \nu\right\rangle}{\sqrt{P_{B}(\mu \mid \beta, \nu)}}$,

where $P_{B}(\mu \mid \beta, \nu)=\left\|\left\langle\mu\left|U^{\dagger}\right| \psi_{\beta}^{\prime}, \nu\right\rangle\right\|^{2}$.

The backward trajectory is specified by the quantum numbers $(\beta, v, \mu)$. However, as in the forward case, we can define the augmented trajectory $\mathcal{X}=(a, \mu, \beta, \nu)$ by introducing the conditional probability $p_{a \mid \beta, v, \mu}=\left|\left\langle\psi_{\alpha} \mid \Phi_{B \mid \beta v \mu}\right\rangle\right|^{2}$. The probability for the augmented backward trajectory will then be given by

$\mathcal{P}_{B}[\mathcal{X}]=p_{a \mid \beta, \nu, \mu} P_{B}(\mu \mid \beta, \nu) p_{\beta}^{\prime} q_{\nu}$.

With the path probabilities (35) and (38), we can now define the entropy production in the usual way, as

$\sigma[\mathcal{X}]=\ln \frac{\mathcal{P}_{F}[\mathcal{X}]}{\mathcal{P}_{B}[\mathcal{X}]}$

By construction, $\sigma$ satisfies a detailed fluctuation theorem ${ }^{40,48,49}$ $\left\langle e^{-\sigma[\mathcal{X}]}\right\rangle=1$

Moreover, similarly to Eq. (36), it follows that $p_{\alpha \mid \beta, v, \mu} P_{B}(\mu \mid \beta, v)=P$ $(\beta, v \mid a, \mu)$, so that

$\sigma[\mathcal{X}]=\ln \frac{p_{\beta \mid a, \mu, \nu} P_{F}(\nu \mid a, \mu) p_{a} q_{\mu}}{p_{\alpha \mid \beta, \nu, \mu} P_{B}(\mu \mid \beta, \nu) p_{\beta}^{\prime} q_{\nu}}=\ln \frac{p_{\alpha} q_{\mu}}{p_{\beta}^{\prime} q_{\nu}}$.

Thus, we see that the conditional terms cancel out. Physically, this means that there is no additional entropic cost in introducing the augmented trajectories, which is a consequence of the fact that the augmentation was done using the eigenstates $\left|\psi_{\beta}^{\prime}\right\rangle$ of $\rho_{S}^{\prime}$. One may also directly verify that $\langle\sigma[\mathcal{X}]\rangle=\Sigma$ is the average entropy production in Eq. (22).
Next, we address the question of how to define stochastic quantities for the two contributions to the entropy production in Eq. (23). That is, we wish to separate

$\sigma[\mathcal{X}]=\sigma_{d}[\mathcal{X}]+\xi[\mathcal{X}]$

such that $\left\langle\sigma_{d}[\mathcal{X}]\right\rangle=\Sigma_{d}$ and $\langle\xi[\mathcal{X}]\rangle=\Xi$. This can be accomplished by augmenting the trajectory once again to include the populations of the system in the energy basis. That is, we define $\tilde{\mathcal{X}}=\{a, \mu, n, \beta, \nu, m\}$, with associated path probabilities $\mathcal{P}_{F(B)}[\tilde{\mathcal{X}}]=\mathcal{P}_{F(B)}[\mathcal{X}] p_{n \mid a} p_{m \mid \beta^{\prime}}^{\prime}$, where we defined the conditional probabilities $p_{n \mid a}=\left|\left\langle n \mid \psi_{a}\right\rangle\right|^{2}$ and $p_{m \mid \beta}=\left|\left\langle m \mid \psi_{\beta}^{\prime}\right\rangle\right|^{2}$.

We then define the stochastic quantities

$\sigma_{d}[\tilde{\mathcal{X}}]=\ln \left(\frac{p_{n} q_{\mu}}{p_{m}^{\prime} q_{\nu}}\right)$

$\xi[\tilde{\mathcal{X}}]=\ln \left(\frac{p_{a} p_{m}^{\prime}}{p_{\beta}^{\prime} p_{n}}\right)$,

where $p_{n}=\left\langle n\left|\rho_{s}\right| n\right\rangle$ and $p_{m}^{\prime}=\left\langle m\left|\rho_{S}^{\prime}\right| m\right\rangle$ are the populations in the energy eigenbasis at the initial and final states [cf. Eq. (19)]. Summing these two contributions immediately yields Eq. (41). Moreover, one may also verify that $\left\langle\sigma_{d}[\tilde{\mathcal{X}}]\right\rangle=\Sigma_{d}$ and $\langle\xi[\tilde{\mathcal{X}}]\rangle=$ 三. Hence, these quantities do indeed represent the stochastic counterparts of the two contributions to the entropy production.

A second glance at Eq. (44) reveals that on the stochastic level, the entropy production $\xi$ due to the loss of coherence is nothing but the change in information between incompatible bases, a quantity sometimes referred to as information gain. ${ }^{50}$ Hence, we arrive at the important conclusion that the incompatibility between classical and quantum entropy production can be traced back, at the stochastic level, to the basis incompatibility of the quantum rules. Indeed, if we rewrite the fluctuation theorem (40) as $\left\langle e^{-\sigma_{d}[\tilde{X}]-\xi[\tilde{X}]}\right\rangle=1$, we can clearly see that due to the presence of coherences, the classical fluctuation theorem that one finds for diagonal initial states is not satisfied. Instead, it must be corrected by the information gain. It is also possible to draw an alternative interpretation in terms of the entropy production due to quantum measurements, as studied for instance in refs. ${ }^{41,48}$ When a measurement is performed in a basis, which commutes with the system's density matrix, no entropy is produced. Non-commuting measurements, on the other hand, have an associated entropy production related to the loss of coherence. This is precisely the content of Eq. (44). No such additional entropy production was generated for the first augmentation that led us to Eq. (35), as in this case there is no information gain since the basis $\left|\psi_{\beta}^{\prime}\right\rangle$ is the basis that diagonalizes $\rho_{s}^{\prime}$.

\section{DISCUSSION}

We have addressed the role played by quantum coherence in determining the behaviour of the entropy production, a fundamental quantifier of thermodynamic irreversibility. By making physically reasonable assumptions on the form of the dynamics undergone by a quantum system and its environment, we have been able to single out the contribution that quantum coherences, a genuine non-classical feature of the state of a given dynamical system, play in quantifying the rate of irreversible entropy production. Such contribution appears to be fully distinct from the one arising from unbalances in the energy eigenbasis of the state of the system, which brings about a classical flavour. Moreover, it can be interpreted in a physically transparent manner as the thermodynamic cost that one has to pay for the destruction 
of coherences that were seeded in the state of the system itself. In turn, our results have interesting consequences for the interpretation of the process of producing entropy as a result of the dynamical generation of correlations (or equivalently coherences) between a quantum system and its environment.

We believe that the theory put forth in this paper may prove a useful step forward towards the setting up of a self-contained framework for the interpretation of thermodynamic irreversibility at the quantum nanoscale, which is still sorely missing despite the key role that entropy production will play in the quantification of the thermodynamic fingerprint of managing quantum dynamics. For instance, it could serve as a starting point for the development of a theory of quantum entropy production in non-equilibrium steady states of systems connected to multiple reservoirs. Or, as a tool for quantifying the contribution of loss of coherence in the operation of finite-time quantum heat engines.

\section{DATA AVAILABILITY}

Data sets were generated or analyzed during the current study.

\section{ACKNOWLEDGEMENTS}

G.T.L. acknowledges the funding from the São Paulo Research agency, under grant number 2016/08721-7. M.P. acknowledges funding from the SFI-DfE Investigator Programme, the EU H2020 Collaborative Project TEQ (grant number 766900), the Leverhulme Trust, and the Royal Society. J.P.S. and G.T.L. acknowledge the Brazilian funding agency CAPES.

\section{AUTHOR CONTRIBUTIONS}

J.P.S. developed the detailed formal calculations in collaboration with G.T.L.; L.C.C., G. T.L. and M.P. conceived the original idea and shaped it with the significant help of J.P. S. All authors contributed to the research and the preparation of the manuscript.

\section{ADDITIONAL INFORMATION}

Competing interests: The authors declare no competing interests.

Publisher's note: Springer Nature remains neutral with regard to jurisdictional claims in published maps and institutional affiliations.

\section{REFERENCES}

1. Onsager, L. Reciprocal relations in irreversible processes. I. Phys. Rev. 37, 405 (1931).

2. Onsager, L. Reciprocal relations in irreversible processes. II. Phys. Rev. 38, 2265 (1931).

3. Machlup, S. \& Onsager, L. Fluctuations and irreversible process. II. Systems with kinetic energy. Phys. Rev. 91, 1512 (1953)

4. Groot de, S. R. \& Mazur, P. Non-Equilibrium Thermodynamics 1st edn, 536 (NorthHolland Physics Publishing, Amsterdam, 1961).

5. Tisza, L. \& Manning, I. Fluctuations and irreversible thermodynamics. Phys. Rev. 105, 1695 (1957).

6. Escher, B. M., de Matos Filho, R. L. \& Davidovich, L. General framework for estimating the ultimate precision limit in noisy quantum-enhanced metrology. Nat. Phys. 7, 406 (2011).

7. Verstraete, F., Wolf, M. M. \& Cirac, J. I. Quantum computation and quantum-state engineering driven by dissipation. Nat. Phys. 5, 633 (2009).

8. Tomé, T. \& De Oliveira, M. C. Entropy production in irreversible systems described by a Fokker-Planck equation. Phys. Rev. E 82, 021120 (2010).

9. Spinney, R. \& Ford, I. Entropy production in full phase space for continuous stochastic dynamics. Phys. Rev. E 85, 051113 (2012).

10. Landi, G. T., Tomé, T. \& de Oliveira, M. J. Entropy production in linear Langevin systems. J. Phys. A 46, 395001 (2013).

11. Schnakenberg, J. Network theory of microscopic and macroscopic behavior of master equation systems. Rev. Mod. Phys. 48, 571 (1976).

12. Tomé, T. \& De Oliveira, M. C. Entropy production in nonequilibrium systems at stationary states. Phys. Rev. Lett. 108, 020601 (2012).

13. Streltsov, A., Adesso, G. \& Plenio, M. B. Colloquium: quantum coherence as a resource. Rev. Mod. Phys. 89, 041003 (2017).
14. Baumgratz, T., Cramer, M. \& Plenio, M. B. Quantifying coherence. Phys. Rev. Lett. 113, 140401 (2014).

15. Oppenheim, J., Horodecki, M., Horodecki, P. \& Horodecki, R. Thermodynamical approach to quantifying quantum correlations. Phys. Rev. Lett. 89, 180402 (2002).

16. Horodecki, M. \& Oppenheim, J. Fundamental limitations for quantum and nanoscale thermodynamics. Nat. Commun. 4, 2059 (2013).

17. Lostaglio, M., Jennings, D. \& Rudolph, T. Description of quantum coherence in thermodynamic processes requires constraints beyond free energy. Nat. Commun. 6, 6383 (2015). arXiv:1405.2188.

18. Lostaglio, M., Korzekwa, K., Jennings, D. \& Rudolph, T. Quantum coherence, timetranslation symmetry, and thermodynamics. Phys. Rev. X 5, 1 (2015).

19. Cwiklinski, P., Studzinski, M., Horodecki, M. \& Oppenheim, J. Limitations on the evolution of quantum coherences: towards fully quantum second laws of thermodynamics. Phys. Rev. Lett. 115, 210403 (2015).

20. Misra, A., Singh, U., Bhattacharya, S. \& Pati, A. K. Energy cost of creating quantum coherence. Phys. Rev. A 93, 052335 (2016).

21. Das, S., Khatri, S., Siopsis, G. \& Wilde, M. M. Fundamental limits on quantum dynamics based on entropy change. J. Math. Phys. 59, 012205 (2017).

22. Korzekwa, K., Lostaglio, M., Oppenheim, J. \& Jennings, D. The extraction of work from quantum coherence. New J. Phys. 18, 023045 (2016).

23. Vacanti, G., Elouard, C. \& Auffeves, A. The work cost of keeping states with coherences out of thermal equilibrium. Preprint at arXiv:1503.01974 (2015).

24. Kammerlander, P. \& Anders, J. Coherence and measurement in quantum thermodynamics. Sci. Rep. 6, 22174 (2016).

25. Francica, G., Goold, J. \& Plastina, F. The role of coherence in the non-equilibrium thermodynamics of quantum systems. Preprint at arXiv:1707.06950 (2017).

26. Brandão, F. G. S. L., Horodecki, M., Oppenheim, J., Renes, J. M. \& Spekkens, R. W. Resource theory of quantum states out of thermal equilibrium. Phys. Rev. Lett. 111, 1 (2013). arXiv:1111.3882.

27. Zurek, W. H. Pointer basis of the quantum apparatus, into what mixture does the wave packet collapse? Phys. Rev. D 24, 1516 (1981).

28. Zurek, W. H. Quantum Darwinism. Nat. Phys. 5, 181 (2009).

29. Alicki, R. \& Lendi, L. Quantum Dynamical Semigroups and Applications 2nd edn (Springer, New York, 2008).

30. Breuer, H.-P. \& Petruccione, F. The Theory of Open Quantum Systems 636 (Oxford Univ. Press, USA, 2007).

31. Gardiner, C. \& Zoller, P. Quantum Noise 3rd edn, 450 (Springer, Berlin, 2004).

32. Callen, H. B. Thermodynamics and an Introduction to Thermostatistics 2nd edn, 493 (Wiley, New York, 1985).

33. Spohn, H. J. Entropy production for quantum dynamical semigroups. Math. Phys. 19, 1227 (1978).

34. Breuer, H.-P. Quantum jumps and entropy production. Phys. Rev. A 68, 032105 (2003).

35. Deffner, S. \& Lutz, E. Nonequilibrium entropy production for open quantum systems. Phys. Rev. Lett. 107, 140404 (2011).

36. de Oliveira, M. J. Quantum Fokker-Planck-Kramers equation and entropy production. Phys. Rev. E 94, 012128 (2016).

37. N. Y. Halpern. in Information and Interaction (eds Durham, I. T. \& Rickles, D.), 135-166 (Springer, 2017).

38. Micadei, K. et al. Reversing the thermodynamic arrow of time using quantum correlations. Preprint at arXiv:1711.03323 (2017).

39. Lindblad, G. Completely positive maps and entropy inequalities. Commun. Math. Phys. 40, 147 (1975).

40. Manzano, G., Horowitz, J. M. \& Parrondo, J. M. R. Quantum fluctuation theorems for arbitrary environments: adiabatic and non-adiabatic entropy production. Phys. Rev. X 8, 031037 (2018).

41. Manzano, G., Horowitz, J. M. \& Parrondo, J. M. Nonequilibrium potential and fluctuation theorems for quantum maps. Phys. Rev. E 92, 1 (2015).

42. Esposito, M., Lindenberg, K. \& Van Den Broeck, C. Second law and Landauer principle far from equilibrium. New J. Phys. 12, 013013 (2010).

43. Brandão, F. G. S. L., Horodecki, M., Ng, N. H. Y., Oppenheim, J. \& Wehner, S. The second laws of quantum thermodynamics. Proc. Natl Acad. Sci. USA 112, 3275 (2015).

44. Strasberg, P., Schaller, G., Brandes, T. \& Esposito, M. Quantum and information thermodynamics: a unifying framework based on repeated interactions. Phys. Rev. X 7, 021003 (2017).

45. Tan, K. C., Kwon, H., Park, C.-Y. \& Jeong, H. A. Unified view of quantum correlations and quantum coherence. Phys. Rev. A 94, 022329 (2016).

46. Park, J. J., Kim, S. W. \& Vedral, V. Fluctuation theorem for arbitrary quantum bipartite systems. Preprint at arXiv:1705.01750 (2017).

47. Dirac, P. A. M. On the analogy between classical and quantum mechanics. Rev. Mod. Phys. 17, 195 (1945).

48. Elouard, C., Herrera-Martí, D. A., Clusel, M. \& Auffeves, A. The role of quantum measurement in stochastic thermodynamics. npj Quant. Inf. 3, 9 (2017). arXiv:1607.02404. 
49. Crooks, G. E. Nonequilibrium measurements of free energy differences for microscopically reversible Markovian systems. J. Stat. Phys. 90, 1481 (1998).

50. Groenewold, H. J. A problem of information gain by quantal measurements. Int. J. Theor. Phys. 4, 327 (1971).

(i) Open Access This article is licensed under a Creative Commons Attribution 4.0 International License, which permits use, sharing, adaptation, distribution and reproduction in any medium or format, as long as you give appropriate credit to the original author(s) and the source, provide a link to the Creative
Commons license, and indicate if changes were made. The images or other third party material in this article are included in the article's Creative Commons license, unless indicated otherwise in a credit line to the material. If material is not included in the article's Creative Commons license and your intended use is not permitted by statutory regulation or exceeds the permitted use, you will need to obtain permission directly from the copyright holder. To view a copy of this license, visit http://creativecommons. org/licenses/by/4.0/.

C) The Author(s) 2019 